\title{
¿Revolución de Facebook? Medios sociales y movimientos sociales durante la Primavera Árabe de Egipto*
}

\author{
Luis Ferando Barón, Ph.D.**
}

\begin{abstract}
Resumen
Activistas y líderes de opinión pública han caracterizado a la Primavera Árabe en Egipto de enero del 2011 como una Revolución de Facebook. Ellos han resaltado el poder intrínseco de los Medios Sociales, que, innegablemente, jugaron un rol importante para el cambio social producido en este proceso revolucionario. Sin embargo, dicho rol, no puede desligarse del contexto socio-político, ni de los desarrollos tecnológicos, ni de las condiciones económicas experimentadas en Egipto en las dos últimas décadas. Este artículo discute el uso de los Medios Sociales, particularmente de Facebook, por parte del Movimiento de la Juventud del 6 de Abril (A6YM por sus siglas en inglés), actor decisivo durante las protestas egipcias. Está basado en un análisis de las noticias de a dos periódicos egipcios y otro estadounidense, entre el 2008 y el 2011. De esta manera, se argumenta que los Medios Sociales: a) funcionaron como mecanismos alternativos para la expresión política y organizacional; b) contribuyeron a la formación y fortalecimiento de la A6YM, además, de la consolidación de las identidades políticas de las juventudes en Egipto. Y finalmente, c)sostiene que la combinación de acciones en la Web y en las calles amplificaron tanto la movilización de colectividades, como también la reacción del Estado en contra de sus opositores.
\end{abstract}

Palabras clave: Facebook, Medios sociales, Movimientos sociales, Primavera árabe, Egipto

* La traducción de este artículo fue realizada por Jacobo Cortés y María Alejandra Peláez. Para esta publicación, el autor revisó y enriqueció su contenido. Revisado y notas a pie de página por Maria A. López.

** Este trabajo es resultado de un proyecto de investigación del TASCHA (acrónimo de Technology \& Social Change Group), Escuela de Información de la Universidad de Washington. Una versión previa de este texto fue publicada en International Review of Information Ethics Vol. 18 (12/2012). 


\section{Introducción}

Periodistas, activistas egipcios e incluso académicos han caracterizado las protestas que comenzaron en Egipto en enero 25, 2011, como una Revolución de Facebook. Este título pone de manifiesto el poder intrínseco de los Medios Sociales ${ }^{1}$ y la potencialidad que el acceso a la WEB puede provocar a los procesos de democratización y cambios sociales. ${ }^{2}$ El uso de TIC (Tecnología de la Información y la Comunicación) cumplió un papel fundamental antes, durante y después de los eventos que se llevaron a cabo en la Plaza de Tahrir en enero y febrero del 2011. No obstante, el rol de los Medios Sociales no puede desconectarse de los contextos socio-políticos regionales e internacionales. Por lo anterior, se debe tener en consideración la historia de las protestas y movimientos sociales en Egipto y la importancia de las organizaciones de las juventudes y sus redes sociales.

El papel de los Medios Sociales no debe estudiarse sin reconocer de antemano los múltiples esfuerzos realizados por las organizaciones musulmanas, en pro de fomentar un movimiento social durante las últimas tres décadas, o los procesos de protesta de los trabajadores, que entre 1998 y el 2009 movilizaron más de dos millones en aproximadamente tres mil trescientas acciones colectivas. Tampoco puede pasarse por alto el surgimiento de las nuevas formas de expresión política, las cuales tienen sus raíces en los Comités de Solidaridad que se propagaron por todo el territorio egipcio, posterior al inicio de la Segunda Intifada en Palestina en octubre del 2000. Los Medios Sociales deben ser considerados, dentro del contexto de las dinámicas regionales, así como las revoluciones de Irán (2009) y Túnez (2010), y las campañas por los derechos humanos lanzadas por organizaciones locales e internacionales en el Medio Oriente y el África del Norte (MENA para su sigla en inglés), y la incesante presión internacional, especialmente proveniente de Estados Unidos y algunos países de la Unión Europea, que buscaba instigar al régimen de Mubarak para llevar a cabo una serie de reformas políticas.

1 Tomado de Kaplan y Haenlein (2010), los medios sociales son considerados en este caso como el grupo de aplicaciones basadas en el servicio de internet, que permiten la creación y el intercambio de contenido generado por un usuario. Estos autores clasifican a los medios sociales en seis categorías: proyectos colaborativos (e.g. wikis); blogs y microblogs (e.g. Twitter); comunidades de contenido (e.g. Youtube); redes sociales (e.g. Facebook); páginas de juegos virtuales (e.g. World of Warcraft), y mundos sociales virtuales (e.g. Second Life).

2 Ver por ejemplo: HTTP://WWW.TECHONOLOGYREVIEW.COM/BLOG/EDITORS/26718/;http:// www.huffingtonpost.com/2011/02/11/egyptfacebook-revolution-wael-ghonim_n_822078.html; http://www.time. com/time/world/article/0.8599,2044142 
Este artículo examinará la relación entre los Medios Sociales y los movimientos sociales, teniendo en cuenta los contextos mencionados antes, con el fin de ofrecer una mirada más compleja del impacto que pudieron tener este tipo de tecnologías en el marco de la Primavera Árabe ${ }^{3}$ de Egipto. Este texto tiene como objetivo poner de manifiesto la manera en que el acceso e interacción con los Medios Sociales, influyó en la formación y desarrollo de las movilizaciones sociopolíticas del A6YM y de los procesos de acción colectiva en Egipto.

Para avanzar en los anteriores propósitos este trabajo realizó un seguimiento exhaustivo a las noticias en inglés relacionadas con las movilizaciones sociales en Egipto entre abril de 2008 y abril de 2011. Tres periódicos, dos egipcios y uno estadounidense, con diferentes enfoques y diversas narrativas fueron utilizados para seguir la situación sociopolítica en Egipto, especialmente en relación con la protesta social: Al-Ahram es un periódico en idioma Inglés semanal emitido por la Organización Al-Ahram. El gobierno egipcio controla un porcentaje significativo de este diario. El Daily News Egypt (DNE) es un periódico independiente, de propiedad privada de Egipto. También se distribuye con el International Herald Tribune (IHT), la versión internacional sólo es en idioma inglés. Es impreso en Egipto y está disponible en el mismo día de su publicación. El New York Times es un periódico estadounidense y su sitio web es uno de los más populares en línea. Para el momento de esta investigación este diario recibía más de treinta millones de visitantes al mes.

La revisión de prensa permite organizar cinco fases del Movimiento Juvenil 6 de Abril. La primera se define por la aparición pública de los primeros miembros del Movimiento. Esta fase fue provocada por la decisión de un grupo de jóvenes de apoyar las protestas de los trabajadores de Mahalla en abril de 2008, mediante el uso de una página de Facebook. La segunda fase está marcada por el proceso de construcción del movimiento A6YM. Esta fase se caracteriza por la decisión del grupo original de involucrar a más jóvenes y organizaciones a través de la expresión de descontento social en lugares y momentos clave. La tercera fase está marcada por la consolidación del movimiento y por la construcción de alianzas con otras organizaciones sociales a nivel nacional. En la

3 La Primavera Árabe, también conocida como la Revolución Árabe, hace referencia a una ola revolucionaria acompañada de manifestaciones y protestas que ocurrieron en el mundo árabe e iniciaron en diciembre de 2010. 
cuarta fase, las alianzas entre organizaciones sociales contrarias al régimen, sumados a una serie de acciones de represión política por parte del Estado condujeron a la construcción de un consenso en torno a un reclamo común: la brutalidad policial. La última fase de este recorrido está representada por las protestas de la Plaza Tahrir, que comenzaron el 25 de enero de 2011, el día de la conmemoración del Día de la Policía.

Para el análisis de la trayectoria de A6YM, se utilizan enfoques alternativos a las Teorías de los Movimientos Sociales (TMS) (e.g. Bayat, 2010; Earl y Kimport, 2011; Melucci, 1994), que desafían las perspectivas más institucionales, como las de la Acción Colectiva, la Movilización de Recursos y la Estructura de Oportunidades Políticas. Estos acercamientos teóricos ayudan a interpretar los resultados del análisis de las noticias de los periódicos analizados. Snow y Soule (2010) ayudan a conceptualizar los movimientos sociales. Bayat (2010), así como Beinin y Vairel, (2011), que confrontan algunos de los principios fundamentales de las Teorías de Movimientos Sociales, ofrecen herramientas conceptuales para comprender las particularidades de las movilizaciones en Oriente Medio y el Norte de África. Earl y Kimport (2011), Garrido (2006) y Melucci (1994), ofrecen herramientas para discutir las relaciones entre los movimientos sociales y las Tecnologías de Comunicación e Información (TIC). Además, proponen retos, tanto para entender los movimientos sociales y como las TIC, en medio de los cambios sociales y tecnológicos de la época contemporánea.

El análisis realizado sugiere que el uso de los Medios Sociales no fue solamente significativo para la creación y consolidación del Movimiento Juvenil 6 de Abril, sino que también jugó un papel importante al momento de convocar a las juventudes a ser parte de las manifestaciones e incentivar su compromiso político con los movimientos sociales. De igual manera, los Medios Sociales promovieron la creación de múltiples escenarios para la acción política, algo que mostró ser efectivo para hacer frente al régimen de Hosni Mubarak y para definir las militares y políticas del régimen.

\section{Teorías de los Movimientos Sociales y las TIC}

Como producto de una extensa revisión y análisis de la literatura sobre movimientos sociales, (Snow y Soule, 2010) definen los movimientos sociales en términos de cinco elementos clave: 1) que son retadores a los defensores o de estructuras o sistemas de 
autoridad existente; 2) que son colectivas en lugar de empresas individuales; 3) que actúan en diversos grados, en las afueras de los acuerdos institucionales u organizacionales existentes; 4) que operan con cierto grado de organización; 5) y por lo general lo hacen con cierto grado de continuidad.

Estos académicos sugieren también un grupo de cinco preguntas clave para la comprensión del carácter y el funcionamiento de los movimientos sociales: la generación de la clase de agravios que contribuyen a la aparición y el funcionamiento de los movimientos sociales; las condiciones contextuales que facilitan o limitan el surgimiento y florecimiento de los movimientos; la tercera y cuarta pregunta se refiere a la participación y las dinámica de los movimientos sociales, y la última indaga sobre las consecuencias y los resultados de los mismos.

Sin embargo, Bayat (2010) ha demostrado que las actuales Teorías de los Movimientos Sociales no pueden explicar efectivamente las complejas dinámicas de resistencia y convulsión dentro de los territorios del Medio Oriente y el Norte de África. Dado el hecho de estas teorías se basan fundamentalmente en las experiencias occidentales y tienen genealogías históricas específicas, difícilmente pueden dar cuenta de las complejidades y particularidades de los movimientos sociales socio-políticos y socio-religiosos de esta zona del mundo (Bayat, 2010).

Beinin y Vairel (2011) también señalan que las teorías de los movimientos sociales han estudiado los movimientos en dicha región con un acercamiento excepcionalista, el cual no explica adecuadamente las complicaciones, producto de los continuos regímenes autoritarios que soslayaron, en diferentes grados a los movimientos sociales. Bayat, por su parte, recalca la importancia de los denominados no-movimientos acciones colectivas de actores no colectivos, además explica cómo los espacios públicos urbanos, especialmente las calles, nos sirven como un elemento esencial para el desarrollo de contiendas. Asimismo, él hace hincapié la relevancia de los espacios de libertad relativa dentro de contextos como el de Egipto (Bayat, 2010).

Además, Bayat afirma que a diferencia de movimientos más tradicionales, como el estudiantil, los cuales requieren un alto grado de organización y estrategia de construcción, los "no-movimientos" pueden aumentar el cambio con su presencia pública. Para el caso de los jóvenes de los no-movimientos, Bayat afirma que estos se caracterizan no 
tanto por lo que los jóvenes hacen, sino por lo que son y cómo están en sus manifestaciones públicas. Así, la identidad de un no-movimiento se basa no tanto en el hacer colectivo como en el ser y estar colectivo, y en las formas de su expresión, son menos protesta colectiva para ser presencia colectiva.

\section{Movimientos y Redes Sociales}

Ya desde comienzos de los años 1990, Melucci (1994) mostraba que los movimientos sociales son los medios de comunicación que hablan a través de la acción: su principal mensaje es que ellos existen y actúan. Sin embargo, las acciones de los movimientos pueden ser vistos como símbolos y como comunicación. "Esto elimina la vieja distinción entre el significado instrumental y expresivo de la acción, en los movimientos contemporáneos los resultados de la acción y la experiencia individual de nuevos códigos tienden a coincidir" (Melucci, 1994:126). Desde esta perspectiva es importante reconocer los movimientos sociales son entramados complejos de individuos, sumado junto con un colectivo de agentes (humanos o no), los cuales constituyen olas de confrontaciones sociales en distintas medidas que abarcan una variedad de formas de manifestación y asociaciones marcadas por su carácter proactivo y de oposición (Escobar, 2008; Melucci, 1994).

Este texto retoma las ideas de Melucci sobre el carácter simbólico y comunicativo de los movimientos sociales, pero también la importancia que éste atribuye a la información, que, desde su punto de vista, se convirtió en un recurso fundamental para los sistemas complejos contemporáneos, como un terreno donde se producen las nuevas formas de poder, la discriminación y el conflicto (Melucci, 1994, 1996). En este sentido, sugiere analizar las relaciones entre las actuaciones (performance), las identidades y las prácticas de comunicación e información en las redes de movimientos sociales, que incluirían los no-movimientos de Bayat.

Por su parte Earl y Kimport (2011) suministran evidencia sobre las maneras en que las TIC han impactado la creación y el desarrollo de los colectivos de acción. Ellos señalan tres aspectos claves: 1). La coexistencia en el tiempo y el espacio de la participación comunal; 2) el carácter efímero, esporádico o persistente de las contiendas; y 3) la certeza sobre la existencia de oposiciones y contrincantes sin la intervención de movimientos sociales organizados. Otros estudios han demostrado la capacidad de la Web para difun- 
dir mensajes en climas hostiles y cómo los grupos activistas han utilizado las TIC para facilitar la comunicación a través de las fronteras y la formación de alianzas (Garrido y Halavais, 2003). En particular, Garrido afirma que las redes de movimientos sociales son discursos desafiantes de poder, proporcionando espacios para que las voces marginadas sean escuchadas mientras que transforma estas voces en un llamado para el cambio social. Además, estos espacios están permitiendo a grupos de personas históricamente explotados, oprimidos y marginados en el centro de la construcción de un proceso de desarrollo alternativo (Garrido, 2006).

Estos cambios no solo han desafiado a los académicos que discuten sobre los movimientos sociales para crear o modificar los enfoques analíticos para entender la acción colectiva, sino que también han estimulado a que diferentes acercamientos teóricos comprendan cómo las TIC están afectando a los movimientos sociales, las prácticas políticas y los procesos de poder (eg. Bennet, Breunig, y Given, 2008; B. Bimber, Flanagin, y Stohl, 200; B.A. Bimber, 2003; Howard, 2010).

\section{Metodología}

Este escrito hace parte de un extenso estudio acerca de los roles que los medios sociales tienen dentro de los movimientos pro-democráticos en Egipto realizado por TASCHA (acrónimo de Technology \& Social Change Group). El estudio más extenso incluye el análisis sobre blogs y grupos de Facebook, tanto en Árabe como en Inglés, medios de comunicación regional (Al Jazeera), y entrevistas con actores de los procesos de movilización política en Egipto. Este texto está basado en el análisis de una muestra que incluye más de 250 artículos sobre las protestas y la movilización de tres periódicos de lengua anglosajona (The Daily News Egypt, Al-Ahram y The New York Times) desde abril del 2008, fecha de origen del Movimiento Juvenil 6 de Abril, hasta abril del 2011, cuando las protestas en la Plaza de Tahrir provocaron la renuncia de Mubarak.

Los periódicos fueron seleccionados con base en los siguientes criterios: a) cubrimiento de las noticias locales e internacionales; b) perspectivas estatales e independientes; c) accesibilidad a través del internet; y d) uso de fuentes de información en inglés. Para la selección de los artículos y el estudio de los mismos, se utilizó una adaptación del marco analítico propuesto por Garrido (2006), que emplea las siguientes categorías: 
a) actores y redes, b) tácticas y estrategias, c) contexto local e internacional, y d) uso de los medios sociales. Siguiendo la perspectiva de (Nastase, Koeszegi, y Szpakowicz, 2007), este estudio desarrolló un análisis de contenido que incluyo los siguientes pasos: a) la recolección de material de las fuentes de prensa; b) la unificación y organización del material textual en unidades para su análisis; c) la clasificación, en la que se desarrollaron y revisaron las categorías pertinentes, respecto a las preguntas de la investigación, a través de un proceso iterativo de análisis; y d) la codificación, en el que se asignaron las unidades a las categorías también a partir de las preguntas de investigación.

\section{Hallazgos}

El análisis de los periódicos seleccionados, permitió definir las siguientes cinco fases del Movimiento de las Juventudes del 6 de Abril (A6YM). Dichas fases deben ser consideradas en relación con los contextos socio-políticos y los mecanismos de comunicación y de medios utilizados por A6YM:

\section{Fase 1: Impulsando una Presencia Pública (Ene - Jun 2008)}

Durante la Primavera de 2008, trabajadores egipcios junto con otros grupos y movimientos de la oposición ${ }^{4}$ hicieron un llamado nacional para llevar a cabo una huelga el día 6 de abril en la compañía textil Ghazl Al-Mahalla. Esta convocatoria se produjo en medio de las elecciones municipales. Asimismo, se produjo en el marco de marchas en protesta por el incremento de los alimentos (Bread Crisis) (El-Fiqi, 2008), y en por los bajos salarios, principalmente de médicos y maestros.

En marzo de 2008, Israa Abdel-Fattah y Ahmed Maher organizaron El Grupo de Huelga del 6 de Abril en Facebook; invitaron amigos y compañeros para apoyar a los trabajadores en su lucha y propagar un mensaje nacional que incitaba a la desobediencia civil. El resultado era inesperado: aproximadamente 77.000 personas se unieron al grupo y se comprometieron, ya sea para manifestarse, o para faltar a sus trabajos el 6 de abril

4 Algunos reportes mencionan la participación del Partido Laborista, el Partido Karama y el Kefaya. Estas fuentes también señalan que la Hermandad Musulmana no apoyaron oficialmente al paro. Vea un ejemplo en: Carr, S. (2008). Activista afirma que las fuerzas de seguridad estatal se movilizan frente a la huelga el seis de abril. The Daily News Egypt, p. :1. RetTomado de: http://www.masress.com/en/dailynews/102028 
(Sarah Carr, 2008b; Singer, 2008). Las protestas del 6 de abril se convirtieron un sello distintivo para la movilización social en Egipto (Sarah Carr, 2008a), y en referente del resurgimiento del movimiento laborista (Sarah Carr, 2008).

Los dos periódicos egipcios informaron sobre las crecientes tensiones en la relación de El Cairo con Washington y sobre la resolución del Parlamento Europeo que critica el historial de derechos humanos de Egipto, y las advertencias que hace sobre la lentitud de las reformas política en Egipto. Mientras la cobertura de Al-Ahram destacó a) las condiciones sociales de las protestas; b) los debates sobre su legalidad en un contexto de una reciente ley que prohibía las manifestaciones públicas en los lugares de culto, y c) la rápida respuesta del gobierno a negociar con los trabajadores; El Daily News Egypt hizo énfasis en a) las dimensiones de la crisis socio-económica; b) las medidas de represión adoptadas por el régimen y c) el terreno ganado por las protestas que realzaron el nivel de antagonismo con el régimen y exponían a la debilidad de la situación oficial (Abdel Meguid, 2008a, 2008b; Sara Carr, 2008; El-Nahhas, 2008; Ezzat 2008; Rady, 2008). Por su parte un artículo del New York Times destacó el papel de las tecnologías (en particular, los mensajes de teléfono celular) que ayudaban a ampliar el descontento de los trabajadores y la creciente voluntad de los trabajadores y profesionales para presionar por sus demandas (Slackman y El-Naggar, 2008).

En esta etapa, Facebook y los mensajes de texto vía móvil jugaron un papel fundamental para fomentar la solidaridad y compromiso con las protestas sociales (especialmente las de los trabajadores). Estas herramientas también fueron utilizadas para estimular la expresión individual de ideas y la creación de espacios de debate político, enfocados en los jóvenes. Aunque aparentemente los participantes de las manifestaciones fueron en buena medida ciudadanos egipcios pertenecientes a sectores privilegiados, los participantes de las protestas hicieron uso de otras herramientas (como folletos, pancartas, y el boca en boca) con el propósito de atraer a otros sectores sociales.

Fase 2: Construyendo un movimiento (Jul 2008 - Jul 2009)

"Estamos debatiendo sobre cómo sacar el mejor provecho de Facebook para organizar actividades”. Entrevista a A. Maher (El-Sayed, 2008) 
A pesar del incremento de las medidas de represión y las constantes amenazas en contra de los grupos de oposición y los "activistas en Facebook", los jóvenes activistas estaban determinados a transformar sus perfiles de Facebook como parte de las luchas sociales (ElSayed, 2008), e involucrar a más jóvenes y organizaciones en las manifestaciones simbólicas organizadas, exponiendo de esta manera, el descontento social en lugares y momentos cruciales. Tanto Israa Abdel-Fattah como Ahmer Maher fueron encarcelados y torturados por las fuerzas de seguridad como resultado de su iniciativa con el grupo de Facebook, denominado "Grupo de Huelga Abril 6". Las detenciones y torturas a estos y otros jóvenes puso al descubierto el alarmante modo de operar de las instituciones frente a la capacidad de movilización de las tecnologías ligadas al Internet- el uso de Facebook y blogs.

Los periódicos analizados, mostraron en este tiempo un intenso debate sobre el derecho a protestar, en el marco del estado de emergencia, que gobernaba desde hace décadas. Este debate se producía no sólo entre las organizaciones sociales y las instituciones del Estado, sino también al interior de las mismas organizaciones sociales. Al-Ahram destacó los planes y decisiones de gobierno para fortalecer los controles y restricciones a la radiodifusión tradicional y el acceso a medios de comunicación social (El-Ghitany, 2008; El-Sayed, 2008a). Mientras tanto El Daily News Egypt, no sólo proporcionó evidencia de blogs y web bloqueados tras el impacto de la campaña en Facebook durante el 6 de abril, sino también ofreció información sobre los arrestos, detenciones y acoso contra los trabajadores y activistas de Internet. Este diario también informó sobre el incremento en las medidas de fuerza adoptadas por el Estado para hacer frente a las, considerados por régimen, como protestas ilegales (Sarah Carr, 2008b).

En esta fase, los medios sociales, particularmente los blogs y las páginas Web, eran usadas como una plataforma para reportar y discutir los eventos en curso y las actividades programadas por los activistas. Facebook jugó un papel importante, no solo en el llamamiento a la los manifestaciones, sino que también permitió crear espacios para generar conversaciones políticas. Por otra parte, éste se convirtió en un referente para la identidad del movimiento social emergente, y uno de los elementos característicos del Movimiento de las Juventudes del 6 de Abril. Posteriormente, Twitter se habría de vincular a las huelgas cuando el periodista James Buck lo usó en el 2008 para reportar su arresto, poco después de las protestas del 6 de abril (Abdoun, 2009). 
Fase 3: Fortaleciendo el movimiento naciente (Ago 2009 - May 2010)

Las protestas y el descontento social con el régimen aumentó durante este periodo de tiempo (Sarah Carr, 2008a; Ree 2008), y el A6YM ganó una posición de influencia en los diferentes escenarios sociopolíticos en Egipto. Este movimiento actuó en calidad de puente entre las distintas organizaciones, mientras que simultáneamente era presionado y acosado por las instituciones estatales (Singer y Samaan, 2008).

\begin{abstract}
"Nosotros empezamos como un fórum para que las personas pudieran interactuar con viejos amigos, subir y compartir fotos, intercambiar mensajes y videos, y matar horas de aburrimiento...después tomó un rumbo diferente en el 2008, particularmente el domingo 6 de abril...los usuarios de Facebook tornaron el popular sitio de entretenimiento en una plataforma con finalidades de activismo político y de la promoción del cambio social' (Abdoun, 2008).
\end{abstract}

Durante este período, tanto Al-Ahram y Daily News informaron sobre el creciente número de protestas. Además, los dos periódicos egipcios y el New York Times destacaron la potencia adquirida por Facebook en manos de los activistas sociales, en particular resaltaban el uso que le daba el A6YM. Los informes del New York Times hicieron hincapié en el aporte de los medios sociales para la organización política y el desarrollo social (Cohen, 2008; Shapiro, 2009). Sin embargo, Al-Ahram destacó los años tensos de las relaciones de Egipto con la administración Bush y la forma en que la elección del presidente Obama abrió una nueva esperanza y un cambio positivo en las relaciones entre USA y Oriente Medio (Ezzat, 2009). El Daily News subrayó la represión a los usuarios de Internet y los informes y las demandas de las organizaciones de derechos humanos locales e internacionales (como la Red Árabe por la Información de Derechos Humanos (ANHRI), Human Rights Watch y Amnistía Internacional y Reporteros sin Fronteras (RSF), que reclamaban la labor de grupos independientes y el respeto de los activistas sociales, así como la libertad de palabra y expresión y del uso de Internet (Abdoun, 2008; Carr, 2009; El-Sayed, 2008b).

En este momento, Facebook se transformó en un objeto/sujeto de las disputas políticas, en un contexto en el que el Estado egipcio incrementaba sus normativas, controles y restricciones. Los medios sociales fueron reconocidos por activistas como herramientas 
de difusión, expresión y acción, las cuales tenían la suficiente capacidad para poner en riesgo al régimen. Los eventos en Irán en julio del 2009 trajeron consigo una mayor exposición al nuevo rol que cumplían los medios sociales en la región, donde los ciudadanos los empleaban para expresar sus opiniones de manera más libre (Abdoun, 2009).

Fase 4: Un reclamo común: La brutalidad Policial (Jun 2010 - Dic 2010)

Para esta etapa, las narrativas de los medios reflejaban un estado de frustración general debido a los resultados de las manifestaciones y las movilizaciones en Egipto (Al Malky,2009). La sociedad en general, particularmente la población juvenil, no tenía expectativas de algún cambio social o político en Egipto (El-Bey, 2010). ${ }^{5}$ Sin embargo, la tortura y posterior muerte de Khaled Saeed a manos de la policía egipcia provocó en un nuevo impulso para las protestas y la atención del público. Después del asesinato de Saeed, dos páginas en Facebook, creadas por activistas, congregaron a aproximadamente de 200.000 simpatizantes en un par de semanas. ${ }^{6}$

Durante el primer semestre de 2012, tanto Al Ahram y Daily News retratan las diferencias y tensiones entre las organizaciones de la sociedad civil que no fueron capaces de crear una fuerza unida contra el régimen de Mubarak. A6YM fue acusado, tanto por el régimen y otras organizaciones sociales de traicionar a Egipto y trabajar en nombre de entidades extranjeras, especialmente de EE.UU. Mientras tanto el New York Times destacó el incremento de las protestas y la represión del gobierno contra los partidos de oposición y en contra de los manifestantes. El NYT también retrató los controles y la vigilancia en Internet y libre flujo de información adoptada por el régimen (AP, 2010). Daily News señaló cómo las respuestas excesivas contra movilización pacífica expusieron la vulnerabilidad del régimen y la pérdida de su credibilidad (Al-Malky, 2010). Todos los periódicos cubrieron eventos ampliamente la tortura y muerte de Saeed, así como las alianzas y las protestas por un cambio social que este evento desencadenó (El-Din, 2010; El-Sayed, 2010; Fahim, 2010).

$5 \quad$ Este artículo está basado en el Reporte del Desarrollo Humano en Egipto (EHDR), el cual indica que la mayoría de las juventudes consideraba que las actividades políticas son inútiles e incapaces de generar alguna diferencia a sus problemas inmediatos.

6 Esta información fue proporcionada en dos artículos publicados por el Daily News Egypt el 20 de junio del 2010. 
El caso de Saeed se convirtió en un flamante impulso para los movimientos juveniles (Elyan, 2010), y también generó más demandas por parte de organizaciones como, Amnistía Internacional, debido a las violaciones de derechos humanos. Por otro lado, el gobierno estadounidense y agentes de la Unión Europea instigaron al gobierno egipcio a implementar una reforma democrática en Egipto. Facebook fue de gran importancia para la construcción de un discurso en contra del uso de la fuerza excesiva por parte de la policía, así como para hacer converger organizaciones de la oposición. Twitter tuvo un rol significativo en cuanto a la diseminación de la información y los detalles de las protestas; los blogs de oposición continuaron confrontando al régimen desde sus múltiples publicaciones.

Fase 5: Ocupando la Plaza Tahrir (Ene - Abr 2011)

"Nosotros nos tomaremos las calles para exigir nuestro derecho a la vida, a la libertad y a la dignidad, y todo lo que nosotros demandamos es que todos se tomen las calles [...] las barreras creadas por el miedo deben romperse" (Sheab, 2011).

En enero del 2011, tres eventos desataron las movilizaciones en Egipto: el incremento del descontento entre los ciudadanos, debido a los resultados de la segunda ronda en las elecciones parlamentarias el 5 de diciembre del 2010; la persistencia de los actos de brutalidad policial, como los actos seguidos a un carro bomba que explotó durante una celebración de año nuevo en una iglesia de Al-Qiddish, en donde se realizó una fuerte campaña liderada por la policía en contra de los manifestantes; finalmente, la revolución tunecina como resultado de una oleada de disturbios sociales y de desobediencia civil. En torno a estos tres sucesos, tanto organizaciones sociales como actores no colectivos, liderados por grupos de jóvenes, incluyendo al A6YM, hicieron sus demandasy llamamientos a la comunidad para ser parte de las huelgas el Día Nacional del Policía, el 25 de enero. Esta convocatoria resultó en la ocupación masiva de la Plaza de Tahrir y la posterior renuncia de Mubarak.

Durante este período los periódicos presentaron a los movimientos juveniles y a las redes de los jóvenes como los actores más importantes de las protestas y destacaron su intenso uso de Facebook, Twitter y YouTube como herramientas clave para los levanta- 
mientos (Mackey, 2011; Shehab, 2011; Tamim, 2011) (Shehab, 2011). Las organizaciones sociales más tradicionales, como los sindicatos de trabajadores o la Hermandad Musulmana, apenas si se mencionan dentro de la cobertura periodística, como parte de los organizadores de las protestas (Shehab, 2011). El Daily News Egypt cubrió con más detalles los enfrentamientos entre manifestantes y fuerzas de seguridad en las calles. Además reportó sobre las numerosas lesiones y muertes causadas en ambos colectividades (Fahmy y Al-A'sar, 2011). Al-Ahram primero subrayó la coalición juventud de organizadores de la protesta y luego animó el debate sobre cómo podrían los movimientos juveniles que provocaron la revolución consolidar su influencia y participar en el proceso político en Egipto (Abdel-Wahab, 2011). The New York Times, en cambio, hizo hincapié en el carácter democrático de los organizadores y las protestas (que calificaron sin un líder visible), e insistió en que no era un movimiento islamista, con miras a no deslegitimar las protestas (Slackman, 2011).

Si la Plaza de Tahrir podría representar el epicentro simbólico de las protestas durante los levantamientos en Egipto, Facebook podría personificar la columna vertebral de las redes creadas por la sociedad civil (mayormente de jóvenes) para expresarse, comunicarse, informarse y movilizarse en contra del régimen de Mubarak. Los medios sociales, en especial Facebook, YouTube, Twitter y los mensajes de texto, fueron fundamentales para la iniciación de las protestas. Estos medios también ayudaron al reporte de los eventos de las movilizaciones en tiempo real, a coordinar las manifestaciones y a construir puntos vinculantes que incentivarán la interacción entre los manifestantes y otros sectores de la sociedad egipcia y de la comunidad internacional. Estos eran instrumentos primordiales para el proceso de difusión de los mensajes que se enfocaban en: a) la habilidad y la oportunidad de los egipcios para producir un cambio social, b) la importancia de la movilización y la toma de las calles, c) la legitimidad de las protestas como un mecanismo para generar reformas políticas, y d) la relevancia de las juventudes como líderes de procesos históricos en Egipto.

\section{Debate y conclusiones}

Después de haber examinado el recorrido de A6YM a través de los lentes de los periódicos, tres hallazgos se logran evidenciar. Los medios sociales no solo ofrecieron mecanismos alternativos para la diseminación de mensajes y acercar a las personas 
dentro de un contexto tan hostil como el que aquí se presenta. En segunda instancia, los medios se convirtieron en un símbolo propio del A6YM y en el proceso de crear su propia identidad, asimismo, los Medios Sociales impactaron de tal manera, que facilitaron la creación, desarrollo y consolidación de este y muchos otros movimientos juveniles.

Uno de los momentos memorables hace alusión a la habilidad que tuvo el A6YM para estructurar y difundir sus discursos para atraer, no solo a jóvenes sino a una audiencia más grande que comprendía movimientos sociales de distinta índole y partidos políticos muy diversos, entre otros. Ellos también recibieron un gran apoyo de organizaciones internacionales (activistas online y organizaciones de defensa de derechos humanos). El movimiento A6YM hizo un excelente trabajo al llevar a los individuos y organizaciones particulares a un campo que concentró una gran variedad de sectores de la sociedad egipcia. El Movimiento también hizo un buen manejo de las palabras y la simbología, haciendo un uso creativo de sus vestuarios y la escogencia de los lugares para realizar sus manifestaciones.

Los miembros del A6YM permiten ver como en las manos de este movimiento estos medios adquieren poder y un rol determinante para estructurar organizaciones y encuentros sociales, con la finalidad de movilizarse en pro de una causa común: derrocar el régimen de Mubarak.

Después de presentar la trayectoria del Movimiento 6 de Abril y de discutir algunas de las interacciones entre este movimiento y los Medios Sociales, surgen una serie de cuestionamientos y desafíos para los estudiosos de estos fenómenos: ¿Es necesario profundizar en los espacios sociales creados por las tecnologías basadas en el uso del internet para la expresión social y la movilización de actores tanto colectivos como no colectivos? También es importante preguntarse acerca de las maneras en que el A6YM y otras organizaciones y diferentes sectores de la sociedad egipcia y la comunidad internacional están diseminando sus narrativas. Igualmente, es válido cuestionarse acerca de la influencia de las redes sociales sobre las dinámicas estatales y sus respuestas frente a los fenómenos sociales. Estas preguntas acompañadas del conocimiento que ahora tenemos de los proceso revolucionarios en Egipto y oros países de la zona, debería llevar a profundizar este tipo de investigaciones desde marcos analíticos y 
metodológicos que respondan a la velocidad, viralidad y capacidad de generar nuevas formas de participación que han generado las fusiones entre movimientos sociales y medios sociales.

\section{Referencias}

Abdoun, S. (2008). The power of Facebook takes new shape in 2008. The Daily News Egypt:1. Retrieved from http://www.masress.com/en/dailynews/114325

Abdoun, S. (2009). Did you tweet today? The Daily News Egypt:1. Retrieved from http://www.thedailynewsegypt.com/did-you-tweet-today.html

Al Malky, R. (2009). The death of youth activism in Egypt? The Daily News Egypt:1. Retrieved from http://www.thedailynewsegypt.com/editorial-the-death-of-youth-activism-in-egypt.html

Bayat, A. (2010). Life as poliTIC : how ordinary people change the Middle East. Stanford, Calif.: Stanford University Press.

Beinin, J., y Vairel, F. (2011). Social movements, mobilization, and contestation in the Middle East and North Africa. Stanford, California: Stanford University Press.

Bennett, L., Breunig, C., y Givens, T. (2008). Communication and Political Mobilization: Digital Media and the Organization of Anti-Iraq War Demonstrations in the U.S. Political Communication, 25(3), 269-289.

Bimber, B., Flanagin, A. J., y Stohl, C. (2005). Reconceptualizing Collective Action in the Contemporary Media Environment. Communication Theory, 15(4), 365-388.

Bimber, B. A. (2003). Information and American democracy : technology in the evolution of political power. Cambridge, UK; New York: Cambridge University Press.

Carr, S. (2008a). April 6 strike kicks off a year of protests. The Daily News Egypt:1. Retrieved from http://www.masress.com/en/dailynews/100950

Carr, S. (2008b). Is it time to sign out of Facebook? The Daily News Egypt:1. Retrieved from http://www.masress.com/en/dailynews/100828

Carr, S. (2008). State security mobilizes ahead of April 6 general strike, says activist. The Daily News Egypt, p. 1. Retrieved from http://www.masress.com/en/dailynews/102028

Earl, J., y Kimport, K. (2011). Digitally enabled social change : activism in the Internet age. Cambridge, Mass.: MIT Press. 
El-Bey, D. (2010). A vision for youth. Al-Ahram Weekly on line:1. Retrieved from: http://weekly.ahram.org.eg/2010/1005/ec6.htm

El-Fiqi, M. (2008). Mission: get bread. Al-Ahram Weekly on line:1. Retrieved from http://weekly.ahram.org.eg/2008/888/ec1.htm

El-Sayed, M. (2008). Virtual poliTIC. Al Ahram:1.

Elyan, T. (2010). April 6join National Association to collect signatures for political reform. The Daily News Egypt:1. Retrieved from http://www.masress.com/en/dailynews/122771

Escobar, A. (2008). Territories of difference : place, movements, life, redes. Durham: Duke University Press.

Garrido, M. I. (2006). The importance of social movements' networks in development communication: lessons from the Zapatista Movement in Chiapas, Mexico.

Howard:N. (2010). The digital origins of dictatorship and democracy: information technology and political Islam. Oxford; New York: Oxford University Press.

Kaplan, A. M., y Haenlein, M. (2010). Users of the world, unite! The challenges and opportunities of Social Media. Bus. Horiz. Business Horizons, 53(1), 59-68.

Latour, B. (2007). Reassembling the social : an introduction to actor-network-theory. Oxford; New York: Oxford University Press.

Melucci, A. (1994). A Strange kind of newness: What's New in New Social Movements? In E. Laraña, H. Johnston y J. R. Gusfield (Eds.), New social movements: from ideology to identity. Philadelphia: Temple University Press.

Nastase, V., Koeszegi, S., y Szpakowicz, S. (2007). Content Analysis Through the Machine Learning Mill. Group Decision and Negotiation, 16(4), 335-346.

Reem , L. (2008). Heated teachers, cool officials. Al-Ahram Weekly on line:1. Retrieved from http://weekly.ahram.org.eg/2008/913/eg4.htm

Shehab, S. (2011). Time for action. Al Ahram:1.

IRIE. International Review of Information Ethics Vol. 18 (12/2012)

Barón, Luis Fernando (2012). More than a Facebook revolution: Social Movements and Social Media in the Egyptian Arab Spring 92

Singer, M. (2008). Court orders release of April 6 youths, but lawyers expect they will be detained longer. The Daily News Egypt:1. Retrieved from http://www.thedaily- 
newsegypt.com/court-orders-releaseof-april-6-youths-but-lawyers-expect-they-will-bedetained-longer.html

Singer, M., y Samaan, M. (2008). April 6 activists stand by detained peers. The Daily News Egypt:1. 